\title{
Attitude towards Mental Illness among Primary Healthcare Providers: A Community-Based Study in Rural China
}

\author{
Zhenyu Ma $\mathbb{D}^{1},{ }^{1}$ Hui Huang, ${ }^{1}$ Guanghui Nie, ${ }^{1}$ Vincent M. B. Silenzio, ${ }^{2}$ and Bo Wei $\mathbb{D}^{1}$ \\ ${ }^{1}$ School of Public Health, Guangxi Medical University, Nanning, China \\ ${ }^{2}$ Injury Control Research Center for Suicide Prevention, Department of Psychiatry, University of Rochester Medical Center, \\ Rochester, USA
}

Correspondence should be addressed to Zhenyu Ma; zhenyu.m@qq.com and Bo Wei; weibogx@163.com

Received 23 May 2018; Revised 28 August 2018; Accepted 10 September 2018; Published 30 September 2018

Academic Editor: Abdelaziz M. Thabet

Copyright (C) 2018 Zhenyu Ma et al. This is an open access article distributed under the Creative Commons Attribution License, which permits unrestricted use, distribution, and reproduction in any medium, provided the original work is properly cited.

\begin{abstract}
Objective. There are no studies that have explored attitudes towards mental illness that are held by rural primary healthcare (PHC) providers. The aim of this study was to conduct evidential and comparative research about attitudes towards mental illness among primary healthcare providers from different mental health service models in China rural communities. Methods. A self-administered questionnaire was conducted with a total of 361 rural primary healthcare providers engaged in mental health service delivery. Results. Total attitude score mark of rural primary healthcare providers shows that most PHC providers still held pessimistic and negative attitude towards mental illness patients. $71.3 \%$ of respondents agreed that "the mental patients often impulsively perform destruction of property"; $72.9 \%$ agreed that "mental patients are burdens to the families and society." There are also positive correlations between attitudes and abilities of primary healthcare providers to mental illness. Conclusion. This study provides baseline evidence that primary healthcare providers in rural China hold negative attitudes towards mental illness. It is critical to improve negative attitudes and understanding about the importance of the management of severe mental illness among rural primary healthcare workers in mental health services. We should take comprehensive methods to enrich primary healthcare providers' professional knowledge about mental illness and eliminate discrimination and inappropriate perception against the mental illness.
\end{abstract}

\section{Introduction}

Integrating mental health services into primary care World Health Organization (WHO) report showed that integrating mental health services into primary care generates good health outcomes at reasonable costs. It is the most viable way of closing the treatment gap and ensuring that people get the mental healthcare they need [1]. That means mental health services should be delivered by the primary healthcare (PHC) providers rather than psychiatrists. Mental health in China is a great public health concern given the large number of patients and huge social and economic implications. In 2009, the new healthcare reform integrated severe mental illnesses into the national primary public health services and stipulated that PHC providers should manage patients with severe mental illness [2]. Essential services at this level include early identification of mental disorders, management of stable psychiatric patients, referral to other levels where required, and promotional and prevention activities. Now the PHC provider is the largest group of mental health service workforce, so their attitudes towards mental illness may affect the quality of mental health services [3].

Guangxi is a less developed area in China with a lack of mental health professionals, especially in rural communities. Due to the shortage and urban-centered unequal distribution of mental health professionals, the improvement and development of community mental healthcare has to rely on the primary healthcare providers, also known as "village doctors" who are usually from the same community and are familiar with the community members in the area [4]. Mental healthcare provision should be switched from traditional psychiatric hospitals-centered pattern to hospitalcommunity based service pattern.

Most previous studies regarding the attitude towards mental illness were conducted in the general population $[5,6]$ and medical students $[7,8]$. However, little is known 
in our own as well as in other cultures about the attitudes PHC providers have about mental illness and those affected. Thus, in this study, firstly we assessed attitudes towards mental illness in a sample of Chinese rural PHC providers. Secondly, we compared the outcomes among two different mental health service models in rural Guangxi: one is hospital-community integrated service model and the other is the psychiatric hospital-centric model. To the best of our knowledge, this is the first study to examine the attitudes towards mental illness among primary healthcare providers who provide the majority of first-line care.

\section{Methods}

2.1. Sample Area Selection and Participants. We selected participants from the two different mental health service model areas in rural Guangxi. The sample area is Liujiang County, with a new hospital-community integrated service model; this program covers 500000 general population in 11 towns. The National Continuing Management and Intervention Program for Psychoses (686 Program) has been developed in Liujiang since 2006. However, Liucheng County is the control area in this study, and its socioeconomic development level and capacity of rural health services are comparable with Liujiang County, and it still follows the traditional hospitalcentric model of community mental health services [4].

We randomly selected 3 towns from Liujiang County according to the socioeconomic status of high, medium, and low. Also, we arranged 3 towns in Liucheng County to compare with Liujiang County in accordance with the socioeconomic situation. There were a total of 6 towns from these 2 sample counties. The participants were all of the 316 primary healthcare providers who deliver mental healthcare in the 6 towns.

2.2. Research Content and Tools. The interview schedule was divided into two parts. Part I included the sociodemographic data: age, sex, education background, job title, income, and willingness to work also collected. Part II included Mental Illness Attitude Questionnaire developed by Beijing Hui Long Guan Hospital [9], which consisted of six subscales: (1) causes of mental illness, (2) violence and aggression of mental illness patients, (3) hope for psychiatric treatments, (4) social value of mental illness patients, (5) problems of avoidance of mental illness patients, and (6) restrictions on mental illness patients. Each subscale has 5 items. The Mental Illness Attitude Questionnaire has 30 items in total, using a 4 -point scale ( $1=$ strongly disagree, $4=$ strongly agree) to measure attitudes towards causes, treatment, and its impact on individuals and society. Add the scores of all the 5 items in each subscale to get a total score ranging from 5 to 20 and then put it into the form of a percentage to the maximum subscale score. Use the numerator of this percentage (25$100 \%)$ as the value of each subscale for interviewee to make statistical analysis. The score of each subscale ranges from 25 to 100. Higher score indicates more agreement with the attitude represented in the subscales. The questionnaire had been tested with high content consistency and test-retest reliability [10].
2.3. Ethics Statement. In order to eliminate possible bias and encourage participants to report their true attitude, the survey was designed as anonymous. The potential risks and benefits of the survey were described by the interviewers. Interviewers were graduate students at the School of Public Health of Guangxi Medical University and attended training courses on research methods and rural mental health services research. The training also included a session on the ethical aspects of human subject research. To compensate for their time, each participant was given a cash amount of RMB 50 (US\$8). The study was approved by the Ethics Committee of the Guangxi Medical University.

2.4. Statistical Analyses. Statistical analysis was conducted using SPSS 19.0. $t$-test, analysis of variance, $\chi 2$ test, Fisher exact test, and Wilcoxon test were performed to examine the differences between two groups. $P<0.05$ was considered with statistically significance.

\section{Results}

3.1. Sample Characteristics. A total of 316 PHC providers in the six towns agreed to participate in the study, and 291 of them completed all interviews. The response rate was $92.1 \%$. 158 completers (54.3\%) were from Liucheng County and 133 (45.7\%) from Liujiang County. The sex distribution in the completers was 170 males (58.4\%) and 121 females (41.6\%). The mean age was $44.6(\mathrm{SD}=1.8)$, and the mean number of working years was 21 (detailed in Table 1).

\subsection{Attitude towards Mental Illness among PHC Providers} in Rural Community. The results showed that most PHC providers still held pessimistic and negative attitude towards mental illness patients. The 4,5, and 6 subscales described the negative attitude: mental illness patients cannot contribute to society; people should avoid mental illness patients and their family members; mental illness patients' social activities should be limited, with the score were between $63.9 \pm 6.9$ and 54.9 \pm 8.4. It can be inferred that PHC providers held different levels of pessimistic and negative attitude. Meanwhile, from the other three subscale scores, the rural community PHC providers also had some misunderstandings to the awareness of mental illness. $t$-test was performed to examine the attitude subscale scores and the difference was not statistically significant $(\mathrm{P}>0.05)$ (detailed in Table 2$)$.

$\chi^{2}$ test was also performed on each of the 30 psychiatric statements in the Mental Illness Attitude Questionnaire (see details in Appendix). Between two counties, there were only three items that showed statistically significant differences, which were item 10 "the violence acts of mental illness patients were as much as the others" $(P=0.011)$, item 12 "mental illness can be treated" $(P<0.001)$, and item 16 "mental illness patients are able to contribute to society" $(P=0.037)$.

We also found high percentage of agreement in some negative attitude statements: $71.3 \%$ of PHC providers agreed that "mental illness patients often impulsively perform destruction of property," $72.9 \%$ agreed that "mental illness patients are burdens to the families and society," etc. It is worth mentioning that over $92 \%$ of PHC providers were 
TABLE 1: Demographic characteristics of PHC providers in two sample counties.

\begin{tabular}{|c|c|c|c|c|}
\hline \multirow[b]{2}{*}{ Demographic Variable } & \multicolumn{2}{|c|}{ Freq. (\%) } & \multirow{2}{*}{$\chi^{2} / t$} & \multirow{2}{*}{$P$} \\
\hline & $\begin{array}{l}\text { Liu Cheng } \\
(\mathrm{N}=158)\end{array}$ & $\begin{array}{r}\text { Liu Jiang } \\
(\mathrm{N}=133)\end{array}$ & & \\
\hline \multicolumn{5}{|l|}{ Sex } \\
\hline Male & $82(51.9 \%)$ & $88(66.2 \%)$ & 6.051 & 0.014 \\
\hline Female & $76(48.1 \%)$ & $45(33.8 \%)$ & & \\
\hline Age & $45.06 \pm 14.18$ & $44.08 \pm 11.47$ & 0.657 & 0.512 \\
\hline \multicolumn{5}{|l|}{ Education } \\
\hline Junior high school or below & $43(27.2 \%)$ & $19(14.3 \%)$ & 14.736 & 0.002 \\
\hline Senior high school & $73(46.2 \%)$ & $90(67.7 \%)$ & & \\
\hline Junior college & $32(20.3 \%)$ & $19(14.3 \%)$ & & \\
\hline College or above & $10(6.3 \%)$ & $4(3.0 \%)$ & & \\
\hline \multicolumn{5}{|l|}{ Job Title } \\
\hline No title & $113(71.5 \%)$ & $100(75.2 \%)$ & 0.512 & 0.774 \\
\hline Primary & $36(22.8 \%)$ & $26(19.5 \%)$ & & \\
\hline Intermediate or above & $9(5.7 \%)$ & $7(5.3 \%)$ & & \\
\hline \multicolumn{5}{|l|}{ Years of Work } \\
\hline Less than 10 years & $40(25.3 \%)$ & $32(24.1 \%)$ & 3.928 & 0.269 \\
\hline $11 \sim 20$ years & $41(25.9 \%)$ & $46(34.6 \%)$ & & \\
\hline $21 \sim 30$ years & $30(19.0 \%)$ & $26(19.5 \%)$ & & \\
\hline More than 30 years & $44(27.8 \%)$ & $26(19.5 \%)$ & & \\
\hline \multicolumn{5}{|l|}{ Major } \\
\hline Clinical Medicine & $82(51.9 \%)$ & $79(59.4 \%)$ & 6.511 & 0.089 \\
\hline Traditional Chinese medicine & $14(8.9 \%)$ & $7(5.3 \%)$ & & \\
\hline Preventive medicine & $21(13.3 \%)$ & $26(19.5 \%)$ & & \\
\hline Others & $36(22.8 \%)$ & $19(14.3 \%)$ & & \\
\hline \multicolumn{5}{|l|}{ Monthly Income } \\
\hline Less than 1000RMB & $97(61.4 \%)$ & $46(34.6 \%)$ & 42.221 & $<0.001$ \\
\hline 1001 2000 RMB & $54(34.2 \%)$ & $49(36.8 \%)$ & & \\
\hline 2001 3000 RMB & $3(1.9 \%)$ & $25(18.8 \%)$ & & \\
\hline More than $3000 \mathrm{RMB}$ & $2(1.3 \%)$ & $13(9.8 \%)$ & & \\
\hline \multicolumn{5}{|c|}{ Willing to provide mental health services } \\
\hline Yes & $126(79.7 \%)$ & $125(94.0 \%)$ & 10.977 & 0.001 \\
\hline No & $28(17.7 \%)$ & $7(5.3 \%)$ & & \\
\hline
\end{tabular}

TABLE 2: The scores of PHC providers' attitude towards mental illness.

\begin{tabular}{|c|c|c|c|}
\hline Attitude subscales & $\begin{array}{l}\text { Liucheng } \\
(\mathrm{n}=158) \\
\overline{\mathrm{x}} \pm \mathrm{s}\end{array}$ & $\begin{array}{l}\text { Liujiang } \\
(\mathrm{n}=133) \\
\overline{\mathrm{x}} \pm \mathrm{s}\end{array}$ & $\begin{array}{c}\text { Total }(\mathrm{n}=291) \\
\overline{\mathrm{x}} \pm \mathrm{s}\end{array}$ \\
\hline (1) Mental illness is caused by biological factors & $67.9 \pm 8.8$ & $67.4 \pm 8.7$ & $67.6 \pm 8.7$ \\
\hline (2) Mental illness patients' behaviors are violent and aggressive & $69.4 \pm 9.2$ & $69.9 \pm 8.1$ & $69.6 \pm 8.7$ \\
\hline (3) Mental illness is incurable & $54.4 \pm 7.7$ & $55.2 \pm 7.0$ & $54.7 \pm 7.4$ \\
\hline (4) Mental illness patients cannot contribute to society & $64.6 \pm 7.0$ & $63.2 \pm 6.8$ & $63.9 \pm 6.9$ \\
\hline (5) People should avoid Mental illness patients and their family members & $54.5 \pm 8.2$ & $55.3 \pm 8.6$ & $54.9 \pm 8.4$ \\
\hline (6) Mental illness patients' social activities should be limited & $60.5 \pm 10.3$ & $58.4 \pm 8.7$ & $59.6 \pm 9.6$ \\
\hline
\end{tabular}

Note: means and standard deviations in the table are the percentages of the answered scores divided by the total score of subscales (the total score of each subscale is 20). The higher the percentage of means, the more agreement with the statements of the subscale. 
TABLE 3: Correlation analysis of attitudes and abilities of the primary healthcare providers to mental illness.

\begin{tabular}{|c|c|c|c|c|c|}
\hline Spearman correlation analysis & $\begin{array}{l}\text { Recognition } \\
\text { ability }\end{array}$ & $\begin{array}{c}\text { Communication } \\
\text { ability }\end{array}$ & $\begin{array}{l}\text { Execution } \\
\text { ability }\end{array}$ & $\begin{array}{l}\text { Learning } \\
\text { ability }\end{array}$ & $\begin{array}{l}\text { Total } \\
\text { score }\end{array}$ \\
\hline (1) Mental illness is caused by biological factors & -0.086 & -0.001 & 0.058 & 0.084 & -0.011 \\
\hline (2) Mental illness patients' behaviors are violent and aggressive & 0.065 & -0.008 & 0.006 & 0.010 & 0.077 \\
\hline (3) Mental illness is incurable & -0.026 & -0.071 & 0.006 & 0.045 & -0.025 \\
\hline (4) Mental illness patients cannot contribute to society & 0.064 & $-0.144^{*}$ & $-0.161^{* *}$ & $-0.201^{* *}$ & $-0.118^{*}$ \\
\hline (5) People should avoid Mental illness patients and their family members & 0.040 & -0.094 & -0.050 & -0.057 & $-0.119^{*}$ \\
\hline (6) Mental illness patients' social activities should be limited & 0.077 & $-0.161^{* *}$ & $-0.147^{*}$ & $-0.129^{*}$ & -0.110 \\
\hline
\end{tabular}

${ }^{* *} P<0.001,{ }^{*} P<0.05$

in favor of "drugs can control the symptoms of mental illness" and "rehabilitation of mental illness is very effective," which indicated that $\mathrm{PHC}$ providers have recognized the importance of psychotropic drugs to control severe mental illness.

\subsection{Attitudes and Abilities of PHC Providers to Mental Illness.} We examined correlations between attitudes and abilities of PHC providers to mental illness (see Table 3). The more agreement of "mental illness patients cannot contribute to society" and "people should avoid mental illness patients and their family members," the lower mental health service capacity the PHC providers had.

\section{Discussion}

The findings suggest that the attitudes of PHC providers were negative towards the mentally ill, no matter whether in hospital-community integrated service model or psychiatric hospital-centric model. Nowadays, in the era of rapid development of technology and civilization, there has been some improvement in tolerance and attitudes. However, there are still many people who have pessimistic and negative attitude towards mental illness, including $\mathrm{PHC}$ providers themselves. Some think that mental disorders cannot be treated, but we know that effective treatments exist and can be successfully delivered in primary care. Some believe that people with mental disorders are violent or unstable and therefore should be locked away, while in fact the vast majority of affected individuals are nonviolent and capable of living productively within their communities. Some evidence shows that different educational level [11], healthcare systems, and cultural differences [12] seem to have an impact on differences in attitudes towards people with mental illness.

Our results found high agreement in subscale "violence and aggression of mental illness patients." $71.3 \%-83.7 \%$ of PHC providers agreed that "mental illness patients often appear impulse, destroy behaviors," "mental illness patients often lose their temper with no reason," and "mental illness patients often appear unexpected impulsive behaviors." The most common response to mental illness is fear of dangerousness. People hold negative attitudes towards mental illness, particularly, a perception that someone with mental illness is dangerous [13]. For example, the typical statements of PHC providers said in our previous qualitative study were
“Frankly speaking, I am afraid of mental disorders' attack. Especially the manic patients. Even though it is dangerous, it is still my duty to contact with them" and "I do not like to contact with mental disorders. They are abnormal and maybe dangerous" [4]. In addition, this study also found that more PHC providers in Liujiang County thought that "the violence acts of mental illness patients were as much as the others," "mental illness can be treated," and "mental illness patients are able to contribute to society." The different attitudes maybe due to the administration of mental illness in Liujiang County were earlier and longer, which have more training and implementary programs. This is also consistent with the findings of another study in China [14], which reported that, after providing free medication, the majority of patients can get control of the illness, some of which can reintegrate or do some simple productive labor. In Liujiang County, some patients could go back to work and farm after free medication. Therefore, PHC providers in Liujiang County have more recognition of the statement that "mental illness patients are able to contribute to society."

The attitudes towards the mental illness patients will greatly affect the practice of PHC providers. Attitude, as a way to assess the psychological constitution, is relatively long-lasting and stable, and it can predict impact behavior to some extent [15]. Positive and correct attitude can make providers care for patients and provide active treatment, health education, and rehabilitation guidance. Negative attitudes towards mental illness are known to be associated with poorer patient functioning and overall client outcomes. Although PHC providers deliver mental health services for mental disorders, their negative attitudes towards mental illness may affect the ways of mental health services, such as passive work and reluctance; this also may affect patients' self-evaluation and treatment compliance, thus reducing the ultimate effects of the treatment $\mathrm{PHC}$ providers provide.

This study presents baseline data from the rural Chinese context that adds to the evidence on PHC providers' attitude to mental illness. Considering the results of the present study, it seems that comprehensive strategies are necessary, to enrich primary healthcare providers' professional knowledge about mental illness and eliminate discrimination and inappropriate perception against mental illness, no matter whether in hospital-community integrated service model or psychiatric hospital-centric model. We recommend that, first of all, the professional ideological education of the 
TABLE 4: Mental Illness Attitude Questionnaire (developed by Beijing Hui Long Guan Hospital).

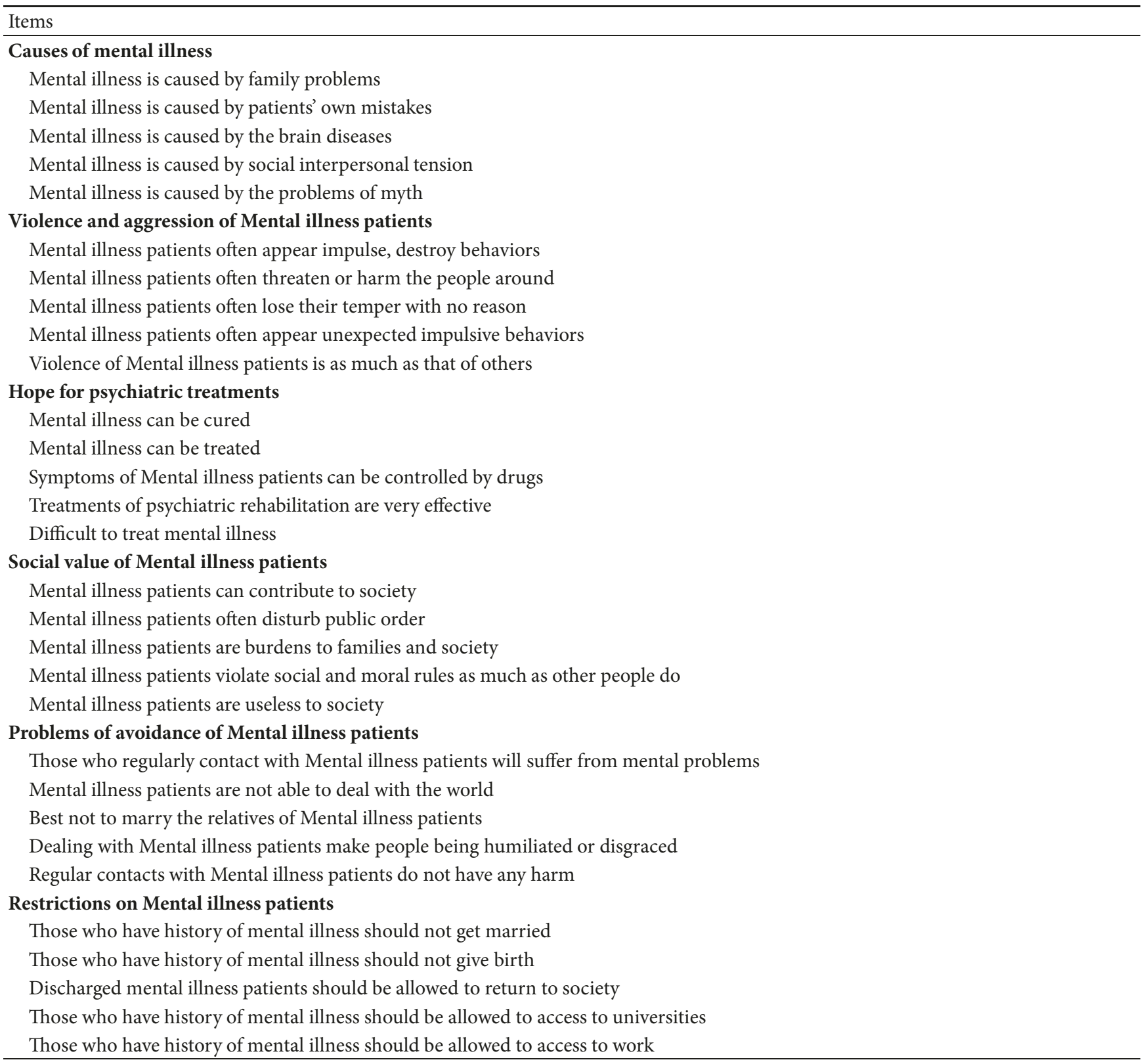

PHC providers should be strengthened, so that they will consciously serve, care, and respect the patients. Second of all, professional continuing education and training should be actively developed, in order to improve the level of mental health awareness for PHC providers [16]. This is critical to improve $\mathrm{PHC}$ providers understanding about the importance of the management of severe mental illness and enrich PHC providers' professional knowledge about mental illness, so that they will have confidence and hope in their future development. There is also a need to weaken and eliminate discrimination and inappropriate perception against mentally ill patients and mental illness by means of community education. To enable understanding and supportive social environment for the mentally ill patients will contribute to the establishment of a good attitude for PHC providers and will be more conducive to the development of community mental health services.

\section{Appendix}

See Table 4.

\section{Data Availability}

The data used to support the findings of this study are available from the corresponding author upon request.

\section{Disclosure}

Zhenyu Ma and Hui Huang are co-first authors. 


\section{Conflicts of Interest}

The authors have no conflicts of interest relevant to this paper to disclose.

\section{Acknowledgments}

This research was supported by Educational Commission of Guangxi province for young college teachers (Grant No. KY2016YB095) and Innovation Project of Guangxi Graduate Education.

\section{References}

[1] World Health Organization and World Organization of Family Doctors, Integrating Mental Health into Primary Care : A Global Perspective, 2008.

[2] Ministry of Public Health, Chinese Mental Health Work Plan (2012-2015), Beijing, China, 2012.

[3] V. Pitt, D. Lowe, S. Hill et al., "Consumer-providers of care for adult clients of statutory mental health services," Cochrane Database of Systematic Reviews, 2013.

[4] Zhenyu Ma, Hu Huang I, Qiang Chen et al., "Mental health services in rural china: A qualitative study of primary health care providers," BioMed Research International, vol. 2015, Article ID 151053, 6 pages, 2015.

[5] T. Högberg, A. Magnusson, M. Ewertzon, and K. Lützén, "Attitudes towards mental illness in Sweden: Adaptation and development of the community attitudes towards mental illness questionnaire," International Journal of Mental Health Nursing, vol. 17, no. 5, pp. 302-310, 2008.

[6] M. C. Angermeyer and S. Dietrich, "Public beliefs about and attitudes towards people with mental illness: A review of population studies," Acta Psychiatrica Scandinavica, vol. 113, no. 3, pp. 163-179, 2006.

[7] B. O. James, J. O. Omoaregba, and E. O. Okogbenin, "Stigmatising attitudes towards persons with mental illness: A survey of medical students and interns from Southern Nigeria," Mental Illness, vol. 4, no. 1, pp. 32-34, 2012.

[8] A. Mas and A. Hatim, "Stigma in mental illness: Attitudes of medical students towards mental illness," Medical Journal of Malaysia, vol. 57, no. 4, pp. 433-444, 2002.

[9] D. Xu, M. Philips, and S. Gao, "Comparison of attitudes on mental illness among doctors and nurses of three psychiatric hospitals," Chinese Mental Health Journal, vol. 14, no. 6, pp. 391394, 2000.

[10] S. Gao and M. Philips, "Attitudes about mental illness of different types of respondents in beijing," Chinese Mental Health Journal, vol. 15, no. 2, pp. 107-109, 2001.

[11] H. F. Mirnezami, L. Jacobsson, and A. Edin-Liljegren, "Changes in attitudes towards mental disorders and psychiatric treatment 1976-2014 in a Swedish population," Nordic Journal of Psychiatry, vol. 70, no. 1, pp. 38-44, 2016.

[12] N. Des Courtis, C. Lauber, C. T. Costa, and K. CattapanLudewig, "Beliefs about the mentally ill: A comparative study between healthcare professionals in Brazil and in Switzerland," International Review of Psychiatry, vol. 20, no. 6, pp. 503-509, 2008.

[13] J. Bennett and R. Stennett, "Attitudes towards mental illness of nursing students in a Baccalaureate programme in Jamaica: A questionnaire survey," Journal of Psychiatric and Mental Health Nursing, vol. 22, no. 8, pp. 599-605, 2015.

[14] L. Liu, X.-L. Chen, C.-P. Ni et al., "Survey on the use of mental health services and help-seeking behaviors in a community population in Northwestern China," Psychiatry Research, vol. 262, pp. 135-140, 2018.

[15] B. D. Fulton, R. M. Scheffler, S. P. Sparkes, E. Y. Auh, M. Vujicic, and A. Soucat, "Health workforce skill mix and task shifting in low income countries: A review of recent evidence," Human Resources for Health, vol. 9, 2011.

[16] Z. Ma, Y. Li, Q. Chen, R. Pan, F. Chen, and Q. Feng, "Evaluation of capability of mental health service among primary health providers in guangxi," Modern Preventive Medicine, vol. 41, no. 20, pp. 3715-3717, 2014. 


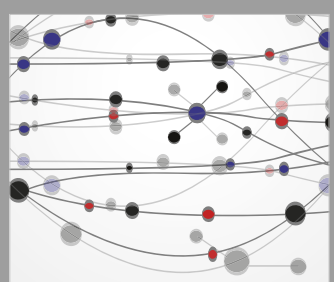

The Scientific World Journal
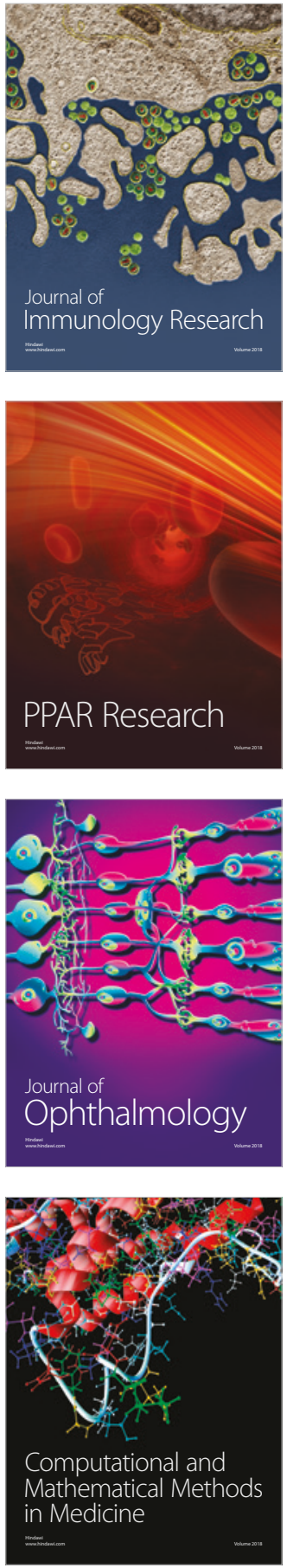

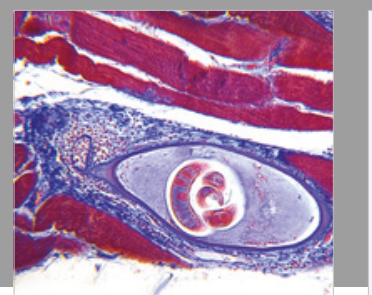

Gastroenterology Research and Practice

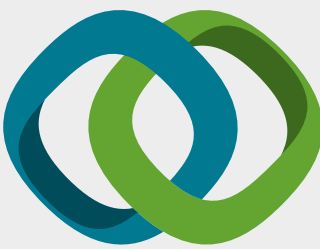

\section{Hindawi}

Submit your manuscripts at

www.hindawi.com
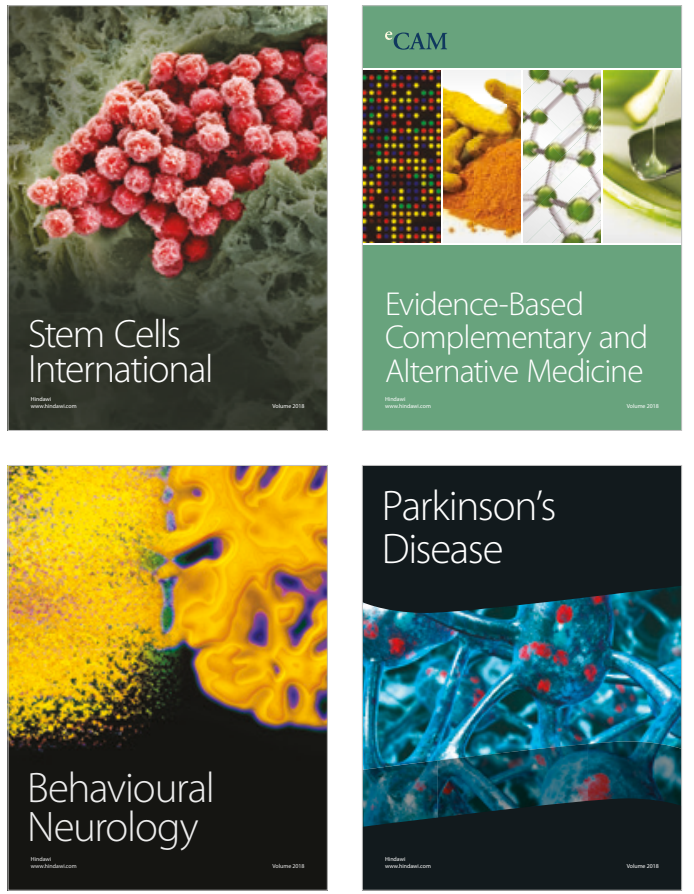

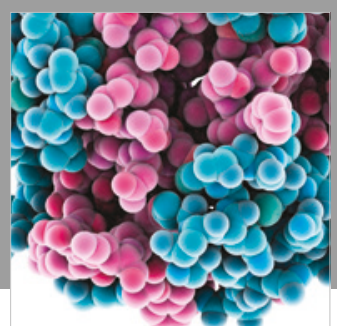

ournal of

Diabetes Research

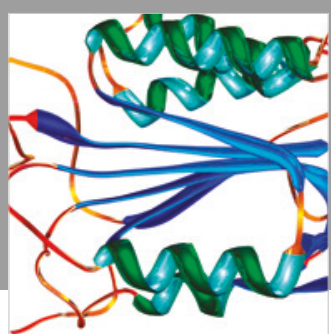

Disease Markers
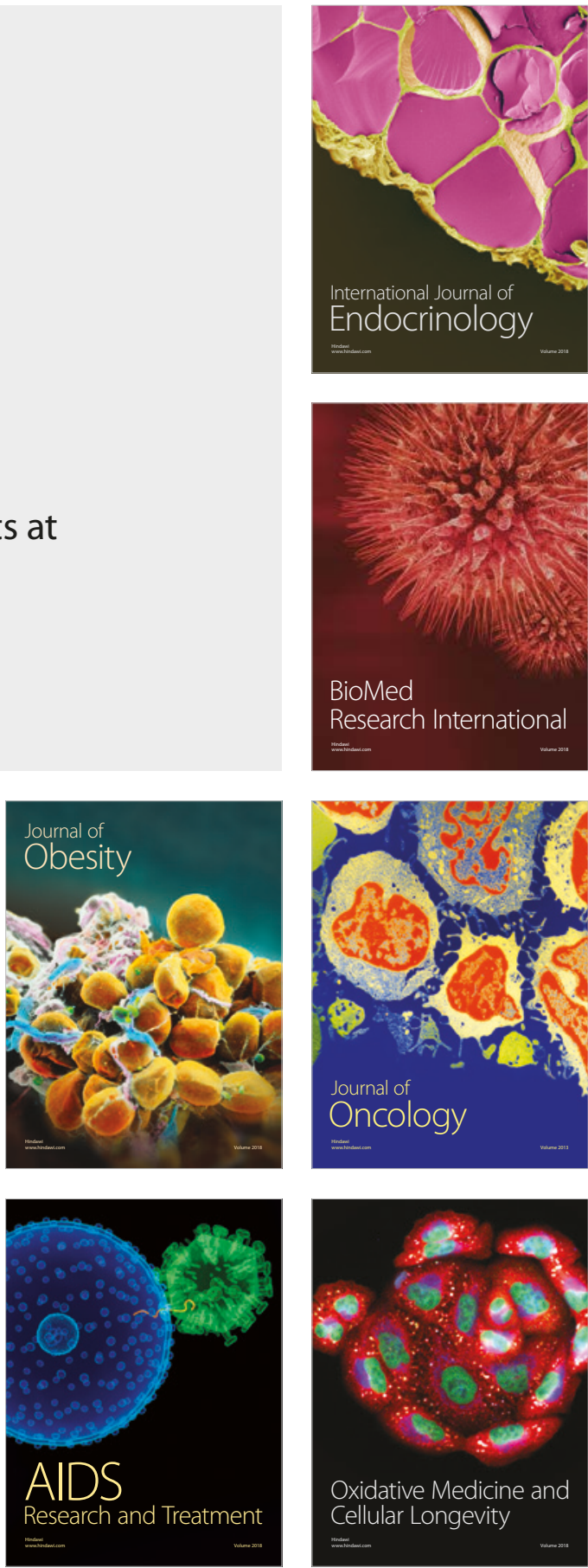